\title{
Even Bonds of Prescribed Directed Parity
}

\author{
Sven Hartmann and C.H.C. Little \\ Massey University, Palmerston North, New Zealand \\ [s.hartmann|c.little] @massey.ac.nz
}

Submitted: Aug 1, 2005; Accepted: Nov 8, 2005; Published: Nov 25, 2005

Mathematics Subject Classification: 05C75

\begin{abstract}
Given a set $S$ of vertices in a graph, the cocycle determined by $S$ is the set of edges joining a vertex in $S$ to a vertex not in $S$. A bond is a minimal non-empty cocycle. We characterise graphs that admit an orientation under which every bond of even cardinality has a prescribed directed parity.
\end{abstract}

\section{Introduction}

In [2], Fischer and Little characterised those graphs that admit an orientation under which every even circuit has directed parity in agreement with a preassigned parity. In other words they assign a parity to every circuit of even length and determine the conditions under which the graph can be oriented so that for every even circuit the parity of the number of edges directed in agreement with a specified sense is equal to the parity assigned to that circuit. In this paper we solve the corresponding problem for even bonds.

Given a graph $G$, let $V G$ and $E G$ denote its vertex set and its edge set, respectively. An edge is called a link if it connects two distinct vertices, and a loop otherwise. Two edges are parallel if they connect the same vertices. By $G[X]$ we denote the subgraph of $G$ induced by a subset $X$ of either $V G$ or $E G$. As long as no confusion arises, we will write $x$ instead of $X$ if $X$ consists of a single element $x$ only.

For any two subsets $S$ and $T$ of $V G$, let $[S, T]$ denote the set of all edges in $E G$ connecting some vertex in $S$ to some vertex in $T$. In particular, $\partial S=[S, V G-S]$ is called a cocycle of $G$. A cocycle $B$ is said to be elementary or a bond if $G$ has a component $C$ such that $B \subseteq E C$ and $C-B$ has just two components. That is, deleting the edges of $B$ from $G$ increases the number of components of $G$ by exactly 1 . It is well known, cf. [1], that a bond is just a minimal non-empty cocycle, and that every cocycle is a sum of disjoint bonds. (The sum of sets is defined as their symmetric difference.)

A bond is even if it consists of an even number of edges, and odd otherwise. An assignment of directed parities to the even bonds of $G$ is a mapping $J$ from the set of even bonds of $G$ into the set $\{0,1\}$. An even bond $B=[S, T]$ in a directed graph is 
$J$-oriented if the number of edges directed from $S$ to $T$ is congruent modulo 2 to $J(B)$. Note that for an even bond $B=[S, T]$ the number of edges directed from $S$ to $T$ is always congruent modulo 2 to the number of edges directed from $T$ to $S$. An orientation of $G$ is $J$ compatible if every even bond of $G$ is $J$-oriented, and $J$-incompatible otherwise. A graph $G$ is $J$-orientable if there exists a $J$-compatible orientation of $G$, and $J$-nonorientable otherwise.

For a subset $A$ of $E G$, let $G<A>$ denote the graph obtained from $G$ by contracting the edges in $E G-A$. We call this graph a contraction of $G$ to $A$. If $E G-A$ contains only a single edge $e$, we say that $G<A>$ is obtained from $G$ by contracting $e$. Note that, when contracting a link $e$, every link parallel to $e$ in $G$ becomes a loop in $G<A>$. Clearly every bond of $G<A>$ is a bond of $G$, too.

Let $H$ be a contraction of $G$. Since bonds of $H$ are also bonds of $G, H$ is $J$-orientable if $G$ is $J$-orientable.

Given adjacent vertices $s$ and $t$ in a graph $G$, the set $R=[s, t]$ containing all edges joining $s$ and $t$ is called a rope of $G$. A graph $G$ is a duplication of a given graph $H$ if $G$ may be obtained from $H$ by adding a non-negative number of edges to each rope of $H$. A duplication is even if the number of edges added to each rope is even.

The objective of this paper is to prove the following characterisation.

Theorem 1. Let $G$ be a graph and $J$ an assignment of directed parities to the even bonds of $G$. Then $G$ is $J$-nonorientable if and only if it has a contraction $H$ which is $J$-nonorientable and an even duplication of a graph in Figure 1.
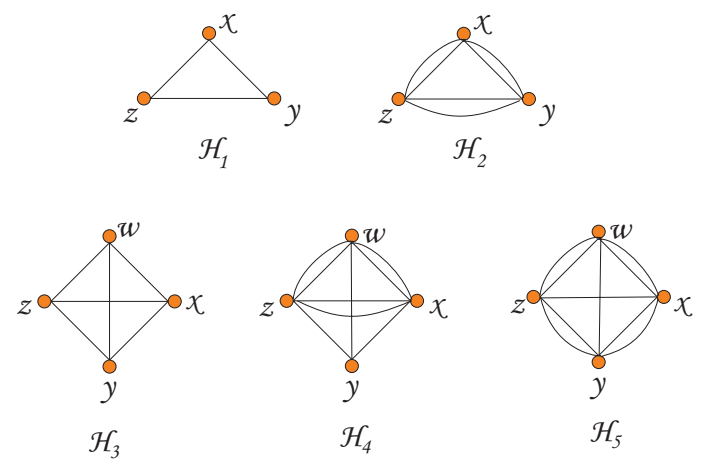
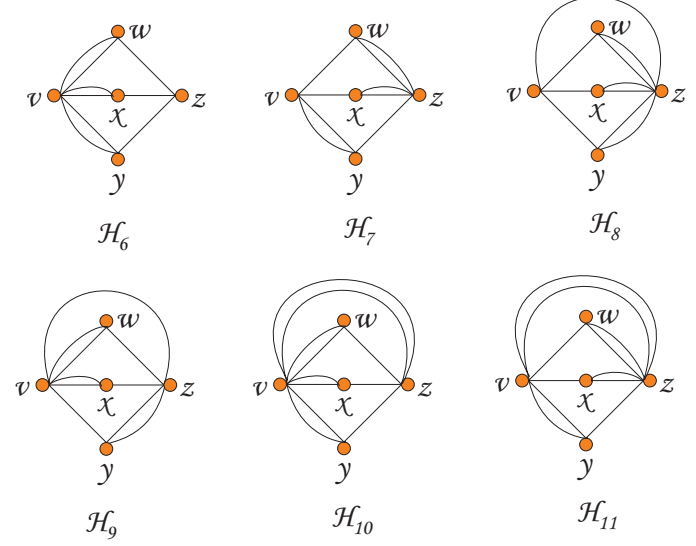

Figure 1: The list.

Let $J$ be an assignment of directed parities to the even bonds of $G$. A set $M$ of even bonds of $G$ is $J$-intractable if, under every orientation of $G$, an odd number of the bonds in $M$ are not $J$-oriented. Note that if the set $M$ of even bonds is $J$-intractable then the symmetric difference of the bonds in $M$ is empty. Clearly, $G$ is $J$-nonorientable if it contains a $J$-intractable set of even bonds. It follows from linear algebra that the converse also holds. 
The following lemma shows that the list of graphs in Figure 1 cannot be reduced any further for the purpose of Theorem 1.

Lemma 2. For each graph $G$ in Figure 1 there is an assignment $J$ of directed parities to the even bonds of $G$ such that $G$ is $J$-nonorientable.

Proof. It suffices to find a non-empty set $M$ of even bonds for each of the graphs in Figure 1 such that the symmetric difference of the bonds in $M$ is empty. The required set $M$ is given for each graph in the table in Figure 2.

\begin{tabular}{c|c} 
graphs & set $M$ of even bonds \\
\hline$H_{1}, H_{2}$ & $\partial x, \partial y, \partial z$ \\
$H_{3}, H_{4}, H_{5}$ & $\partial\{w, x\}, \partial\{w, y\}, \partial\{w, z\}$ \\
$H_{6}, H_{7}, H_{8}, H_{9}, H_{10}, H_{11}$ & $\partial v, \partial\{w, z\}, \partial\{x, z\}, \partial\{y, z\}$
\end{tabular}

Figure 2: Sets of even bonds in the graphs on the list.

\section{Bonds}

In this section we assemble further terminology and preliminary lemmas to be used later on in the proof of Theorem 1. A digon is a duplication of the complete graph $K_{2}$, while a trigon is a duplication of $K_{3}$. We record the following simple observation for further reference.

Lemma 3. $B$ is a bond of a graph $G$ if and only if $G<B>$ is a digon.

Proof. The statement follows immediately from the definition.

A cocycle $[S, T]$ separates two disjoint non-empty subsets $S^{\prime}$ and $T^{\prime}$ of $V G$ if $S^{\prime} \subseteq S$ and $T^{\prime} \subseteq T$.

Lemma 4. Let $H_{1}$ and $H_{2}$ be two vertex-disjoint connected subgraphs of a connected graph $G$. Then there is a bond of $G$ which separates $V H_{1}$ and $\mathrm{VH}_{2}$.

Proof. Let $S$ consist of all vertices accessible in $G$ from vertices in $V H_{1}$ without passing through vertices in $\mathrm{VH}_{2}$. As $G$ is connected, the vertices in $V G-S$ must be accessible in $G$ from vertices in $V H_{2}$ without passing through vertices in $V H_{1}$. Since $H_{1}$ and $H_{2}$ are connected, both $G[S]$ and $G[V G-S]$ are connected, and $[S, V G-S]$ is the desired bond.

The following lemma shows that every bond of a connected subgraph may be extended to a bond of the whole graph by adjoining only edges that are not in the subgraph.

Lemma 5. Let $H$ be a connected subgraph of a graph $G$, and $B$ a bond of $H$. Then there is a bond $B^{\prime}$ of $G$ such that $B^{\prime} \cap E H=B$. 
Proof. By definition $H-B$ has exactly two components $H_{1}$ and $H_{2}$. Further, $H$ is contained in a component $C$ of $G$. By Lemma 4, $C$ has a bond $B^{\prime}=[S, V C-S]$ separating $V H_{1} \subseteq S$ and $V H_{2}$, and this bond is also a bond of $G$. By construction, we have $B \subseteq B^{\prime}$. Every edge in $E H-B$ is either in $H_{1}$ and thus in $G[S]$, or in $H_{2}$ and thus in $G[V C-S]$. In each case, the edge is not in $B^{\prime}$. This concludes our proof.

Corollary 6. Every link in $G$ belongs to some bond of $G$.

\section{Bond-connected graphs}

A cut-set of a connected graph $G$ is a set $S \subseteq V G$ of vertices such that the subgraph $G[V G-S]$, derived from $G$ by removing the vertices in $S$, is no longer connected. If, in particular, a cut-set $S$ consists of a single vertex $v$ only, $v$ is called a cut-vertex of $G$. A connected graph $G$ is $k$-connected if it has at least $k+1$ vertices and does not contain a cut-set of cardinality $k-1$, cf. [1]. It is well known that a graph with at least 3 vertices is 2-connected if and only if any two links belong to a common circuit.

A connected graph $G$ is bond-connected if for every bipartition $\left\{A_{1}, A_{2}\right\}$ of $E G$ there is a bond meeting both $A_{1}$ and $A_{2}$. Clearly, bond-connected graphs cannot have loops.

Lemma 7. Let $G$ be a graph with at least 3 vertices and without loops. G is bond-connected if and only if $G$ is 2-connected.

Proof. Suppose $G$ is bond-connected, and thus connected. Suppose $G-\{v\}$ is not connected for some vertex $v$. Let $C$ be a component of $G-\{v\}$. Choose $A_{1}=E G[V C \cup\{v\}]$ and $A_{2}=E G-A_{1}$. Since $G$ is bond-connected there is a bond $B=[S, V G-S]$ meeting both $A_{1}$ and $A_{2}$. Let $B$ contain an edge $e_{i} \in A_{i}$ joining vertices $s_{i}$ and $t_{i}$ with $s_{i} \in S$ and $t_{i} \in V G-S$ for each $i \in\{1,2\}$. Since $G$ is connected and $B$ is a bond, both $G[S]$ and $G[V G-S]$ are connected. Without loss of generality we may assume that $v \in S$. Then, $G$ has no path joining $t_{1}$ to $t_{2}$ without passing through $v$. This, however, contradicts the connectedness of $G[V G-S]$ and proves $G$ to be 2-connected.

Suppose $G$ is 2-connected, and thus connected. Let $\left\{A_{1}, A_{2}\right\}$ be a bipartition of $E G$. Choose a link $e_{i} \in A_{i}, i=1,2$. Then there is a circuit $C$ of $G$ containing both $e_{1}$ and $e_{2}$. Clearly $\left\{e_{1}, e_{2}\right\}$ is a bond of $G[C]$. By Lemma 5 there is a bond $B$ of $G$ containing $e_{1}$ and $e_{2}$. This proves $G$ to be bond-connected.

Corollary 8. The following three propositions are equivalent for a connected graph $G$ :

1. $G$ is bond-connected.

2. $G$ is a singleton, a digon or a 2-connected graph without loops.

3. $G$ has neither a cut-vertex nor a loop.

Proof. The corollary follows immediately from Lemma 7.

Lemma 9. A graph $G$ is bond-connected if and only if any two of its edges belong to a common bond. 
Proof. Suppose $G$ is bond-connected, and note that all edges in a bond-connected graph are links. The statement is trivial if $G$ has fewer than 3 vertices. Otherwise, let $e_{1}$ and $e_{2}$ be distinct links in $G$. Since $G$ is 2-connected, there is a circuit $C$ containing $e_{1}$ and $e_{2}$. Clearly, $\left\{e_{1}, e_{2}\right\}$ is a bond of the induced subgraph $G[C]$. The claim now follows from Lemma 5. Conversely, if any two edges of $G$ belong to some bond, $G$ is clearly bond-connected.

Lemma 10. Let $G<A>$ be bond-connected, and let $B$ be a bond of $G$ that meets $A$. Then $G<A \cup B>$ is bond-connected.

Proof. The statement holds if $A \subseteq B$, and so we may assume that $A-B \neq \emptyset$. Let $\{X, Y\}$ be a bipartition of $A \cup B$. If $A \subseteq X$ or $A \subseteq Y$, then $B$ meets both $X$ and $Y$. Otherwise $X \cap A \neq \emptyset$ and $Y \cap A \neq \emptyset$ and, in this case, some bond in $G<A>$ meets $X$ and $Y$ since $G<A>$ is bond-connected. This bond is also a bond of $G<A \cup B>$.

\section{Vertex splits and bond-connected graphs}

Given adjacent vertices $s$ and $t$ in a graph $G$, let $R=[s, t]$ be the rope of $G$ containing all edges joining $s$ and $t$. The graph $H=G<E G-R>$ obtained from $G$ by contracting the rope $R$ to a single vertex $v$ is called a rope contraction of $G$, while $G$ is called a vertex split of $H$, and said to be obtained from $H$ by splitting $v$ into $s$ and $t$.

Since $G-\{s, t\}=H[V H-v]$, a vertex $w \in V G \cap V H$ is a cut-vertex of $G$ if and only if it is a cut-vertex of $H$. Further, $v$ is a cut-vertex of $H$ if and only if $\{s, t\}$ is a cut-set of $G$. Moreover, if $s$ itself is a cut-vertex of $G$ then $v$ is a cut-vertex of $H$ or $t$ is incident only with edges in $R$. We call $G$ a proper vertex split of $H$ if $v$ is the only vertex in $H$ or if both $s$ and $t$ are incident in $G$ with some edge not in $R$. In the latter case, $v$ is a cut-vertex of $H$ if $R$ is a bond of $G$.

Lemma 11. Let $R$ be a rope of $G$, and let $H=G<E G-R>$ be bond-connected. Then $G$ is bond-connected if and only if $G$ is a proper vertex split of $H$.

Proof. The claim is true if $H$ has only a single vertex. Otherwise suppose $G$ is a bondconnected graph obtained from $H$ by splitting a vertex $v$ of $H$ into $s$ and $t$. Considering the bipartition $\{R, E H\}$ of $E G$, we find that $R$ is not a bond of $G$ itself and thus both $s$ and $t$ are incident in $G$ with some edge not in $R$. Hence, $G$ is a proper vertex split of $H$.

Conversely, suppose $G$ is a proper vertex split of $H$ obtained by splitting a vertex $v$ of $H$. Let $\left\{A_{1}, A_{2}\right\}$ be a bipartition of $E G$. If $A_{1} \nsubseteq R$ and $A_{2} \nsubseteq R$, then by the hypothesis there is a bond of $H$ meeting both $A_{1}$ and $A_{2}$. This bond is also a bond of $G$. Otherwise, we note that every rope in a graph is a subset of some bond. So there is a bond of $G$ containing $R$. Since $H$ is bond-connected, $v$ is not a cut-vertex of $H$ and thus $R$ is not a bond of $G$ itself, but a proper subset of some bond. This proves $G$ to be bond-connected.

Remark 12. On the other hand, if $G$ is a bond-connected graph obtained from $H$ by splitting a vertex $v$ into $s$ and $t$, then $v$ is the only possible cut-vertex of $H$. Thus, $H$ is bond-connected if and only if $\{s, t\}$ is not a cut-set of $G$. 
Lemma 13. Let $G$ be bond-connected, and let $A$ be a non-empty proper subset of $E G$ such that $G<A>$ is bond-connected. Let $B$ be a bond of $G$ meeting both $A$ and $E G-A$, and let $S$ be a minimal non-empty subset of $B-A$ which is the intersection with $B-A$ of a bond of $G<A \cup B>$. Then $G<A \cup S>$ is a proper vertex-split of $G<A>$.

Proof. Since $G<A>$ is bond-connected it has no loops. Therefore each rope of $G<A \cup S>$ is a subset of $A$ or of $S$. Let $R=[s, t]$ be a rope of $G<A \cup S>$ such that $S$ contains $R$, and suppose there is a vertex $u$ of $G<A \cup S>$ incident with an edge $e$ in $S$ and different from $s, t$. Note that $e$ must be a link since $S$ includes every rope of $G<A \cup B>$ it meets. Let $X$ be the set of links in $G<A \cup S>$ incident with $u$. Then, $X$ is a cocycle of $G<A \cup S>$ and thus a cocycle of $G<A \cup B>$. Hence some subset $D$ of $X$ is a bond of $G<A \cup B>$ containing $e$. Its intersection with $B-A$ contains $e \in S$ but not $R \subseteq S$. Thus $D-A$ is a proper non-empty subset of $S$ contradicting the minimality of $S$. Therefore $G<A \cup S>$ is a vertex-split of $G<A>$. It is proper since the non-empty proper subset $S$ of $B$ cannot be a bond of $G<A \cup S>$.

Lemma 14. Let $G$ be bond-connected, and let $A$ be a non-empty proper subset of EG such that $H=G<A>$ is bond-connected. Then $G$ has a rope $R \subseteq E G-A$ such that $G<E G-R>$ is bond-connected.

Proof. Let us call a rope $R=[s, t]$ bad if $\{s, t\}$ is a cut-set of $G$, and good otherwise. By Remark 12, it suffices to show that there is a good rope in $E G-A$. Suppose not. As $H$ has no loops, there is no rope of $G$ meeting both $A$ and $E G-A$. Choose a rope $R=[s, t] \subseteq E G-A$. Let $C_{0}, C_{1}, \ldots, C_{k}$ be the components of $G-\{s, t\}$. Since $H$ has no cut-vertex, we may assume without loss of generality that $A$ is a subset of $E G\left[V C_{0} \cup\{s, t\}\right]$. Among the remaining components, let $C_{1}$ be one with the smallest number of edges. We may assume that $R$ is chosen to minimise the cardinality of $E C_{1}$.

We claim that $E C_{1}$ is empty. Suppose there is some rope $Q=[x, y] \subseteq E C_{1}$. By assumption, $Q$ is in $E G-A$ and bad. Since $G-\{x, y\}$ is not connected there is a component of $C_{1}-\{x, y\}$ having no vertex adjacent to $s$ or $t$. Therefore $G-\{x, y\}$ has a component whose edge set is disjoint from $A$ and is a proper subset of $E C_{1}$. This contradicts the choice of $R$ and $C_{1}$.

Hence, $C_{1}$ consists of a single vertex $w$ which is adjacent to both $s$ and $t$. The rope $[s, w]$ is good and in $E G-A$ contradicting the assumption. This proves the lemma.

Corollary 15. Every bond-connected graph $G$ with at least 2 vertices has a rope $R$ such that $G<E G-R>$ is bond-connected.

Proof. The corollary is clear if $G$ has exactly 2 vertices. Otherwise it follows from Lemma 14 when choosing $A$ to be a bond of $G$, that is, $H$ to be a digon.

\section{Proof of the main theorem}

Let $G$ be a graph, let $J$ be an assignment of directed parities to the even bonds of $G$, and assume that $G$ is minimally $J$-nonorientable with respect to the contraction of a rope. 
Hence, $G$ is bond-connected. By Corollary $15, G$ has a rope $R$ such that $H=G<E G-R>$ is bond-connected. Since $H$ is $J$-orientable, we may choose a $J$-compatible orientation of $H$, and extend it to an orientation of $G$. Since $G$ is $J$-nonorientable, there exist two even bonds $A$ and $B$ of $G$ containing $R$ such that just one of them has the directed parity prescribed by $J$.
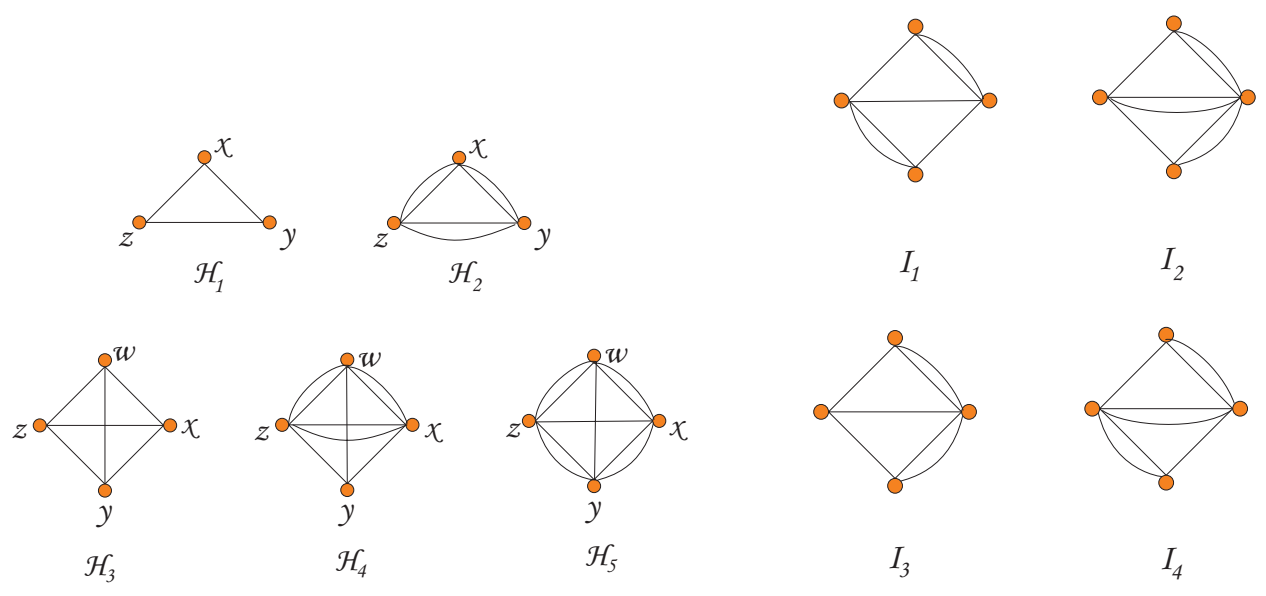

$I_{1}$

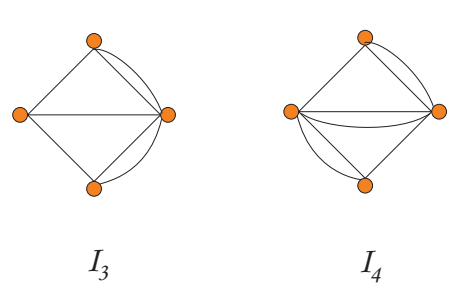

Figure 3: The preliminary list.

Lemma 16. The even bonds $A$ and $B$ can be chosen so that $G<A \cup B>$ is an even duplication of a graph in Figure 3.

A proof of Lemma 16 is given in the next section.

If $G<A \cup B>$ is an even duplication of one of the duplications of $K_{3}$ or $K_{4}$ in Figure 3, we have completed the proof of our main theorem as these graphs also appear in the list for our main theorem. (See Figure 1.) Henceforth, we assume that there is no choice of $R$, and of the even bonds $A$ and $B$ as singled out above, such that $G\langle A \cup B>$ is an even duplication of one of the duplications of $K_{3}$ or $K_{4}$ in Figure 3. By Lemma 16, we nevertheless find even bonds $A$ and $B$ such that $G<A \cup B>$ is an even duplication of one of the duplications of $K_{4}-e$ in Figure 3. In the sequel, we suppose that the rope $R$, and the even bonds $A$ and $B$ are chosen so that $A \cap B$ is minimised.

By Lemma 16, $G<A \cup B>$ is just a duplication of $K_{4}-e$ where the two non-adjacent vertices have odd degree. (See Figure 4.) Note that $G<A \cup B>$ is bond-connected. If $G<A \cup B>$ is an even duplication of $I_{1}$ or $I_{2}$, its even bonds are $\partial x$ and $\partial v$, and its odd bonds are $\partial u, \partial w, \partial\{u, x\}, \partial\{w, x\}$. Hence $A$ and $B$ are $\partial x$ and $\partial v$. If $G<A \cup B>$ is an even duplication of $I_{3}$ or $I_{4}$, its even bonds are $\partial\{w, x\}$ and $\partial\{u, x\}$, and its odd bonds are $\partial u, \partial w, \partial x, \partial v$. Hence $A$ and $B$ are $\partial\{w, x\}$ and $\partial\{u, x\}$.

Remark 17. $A+B$ is the sum of two disjoint odd bonds of $G<A \cup B>$, namely $\partial u$ and $\partial w$. 


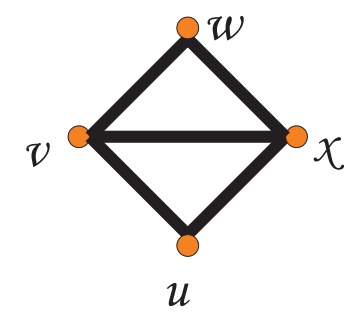

Figure 4: The graph $G<A \cup B>$. Ropes are illustrated by fat black lines. The vertices $u$ and $w$ have odd degree.

The graph $H=G<E G-R>$ is bond-connected. Therefore, it has a bond $Z$ that meets $\partial u$ and $\partial w$. The only bonds of $H<(A \cup B)-R>=G<(A \cup B)-R>$ are $\partial u$ and $\partial w$. Hence, $Z$ meets $E H-(A \cup B)$, too. That is, $Z$ is a bond of $H$ that meets $\partial u, \partial w$ and $E H-(A \cup B)$. Among all bonds $Z$ of $H$ that meet $\partial u, \partial w$ and $E H-(A \cup B)$, choose one that minimises $Z-(A \cup B)$.

Lemma 18. $Z, Z+\partial u, Z+\partial w$, and $Z+\partial u+\partial w$ are bonds of $H$ such that each of them meets $\partial u, \partial w$ and $E H-(A \cup B)$.

Proof. It is easy to check that each of the cocycles $Z+\partial u, Z+\partial w$ and $Z+\partial u+\partial w$ meets $\partial u, \partial w$ and $E H-(A \cup B)$. So it remains to prove that these cocycles are indeed bonds. Assume $T=Z+\partial u$ is not a bond and, therefore, the disjoint union of two or more bonds of $H$. Neither $Z$ nor $\partial u$ is among these bonds. Rather, each of these bonds meets $\partial u$ and $E H-(A \cup B)$. Moreover, one of these bonds, say $Z^{\prime}$, meets $\partial w$, too, since $Z$ meets $\partial w$ while $\partial u$ does not. Thus $Z^{\prime}$ meets $\partial u, \partial w$ and $E H-(A \cup B)$, and $Z^{\prime}-(A \cup B) \subset Z-(A \cup B)$ since $T$ is the disjoint union of $Z^{\prime}$ and at least one further bond meeting $E H-(A \cup B)$. These results contradict the choice of $Z$.

We conclude that $Z+\partial u$ is a bond of $H$. Similarly we prove that $Z+\partial w$ and $Z+\partial u+\partial w$ are bonds.

Let $Z_{1}, Z_{2}$ be the even bonds among $Z, Z+\partial u, Z+\partial w$, and $Z+\partial u+\partial w$. The following observation is an immediate consequence of Lemma 18.

Corollary 19. $G=G<A \cup B \cup Z_{1} \cup Z_{2}>=G<A \cup B \cup Z>$.

Proof. $A, B, Z_{1}$ and $Z_{2}$ are even bonds of $G$ and satisfy $A+B=\partial u+\partial w=Z_{1}+Z_{2}$. As only one of these bonds does not have the directed parity assigned by $J$, we find that $\left\{A, B, Z_{1}, Z_{2}\right\}$ is a $J$-intractable set of even bonds of $G$. Hence $G<A \cup B \cup Z_{1} \cup Z_{2}>$ is $J$-nonorientable, and we find that $G=G<A \cup B \cup Z_{1} \cup Z_{2}>$. Finally, the bonds $Z$, $Z+\partial u, Z+\partial w$, and $Z+\partial u+\partial w$ coincide on their intersection with $E G-(A \cup B)$.

Let $S$ be a minimal non-empty subset of $E G-(A \cup B)$ which is the intersection with $E G-(A \cup B)$ of a bond of $G=G<A \cup B \cup Z>$. By Lemma 13, $G<A \cup B \cup S>$ is a proper vertex split of $G<A \cup B>$ and $S$ is therefore a rope of $G<A \cup B \cup S>$. Further, by Lemma 11, $G<A \cup B \cup S>$ is bond-connected. 
Lemma 20. $H$ has no bond $Y$ with $S \subset Y \subset S \cup \partial u$ or $S \subset Y \subset S \cup \partial w$.

Proof. Suppose there is a bond $Y$ such that $S \subset Y \subset S \cup \partial u$. Let $T$ be the cocycle $Z+Y$. This cocycle meets $\partial w$ and therefore includes a bond $Z^{\prime}$ that meets $\partial w$ necessarily in an edge of $Z$. But $Z^{\prime} \neq Z$ since $Z^{\prime} \cap S=\emptyset$. Hence $Z^{\prime}$ meets $T-Z \subseteq Y$. As $Z^{\prime} \cap S=\emptyset$ it follows that $Z^{\prime} \cap \partial u \neq \emptyset$. Thus $Z^{\prime}$ meets $\partial u$ and $\partial w$, and $Z^{\prime}-(A \cup B) \subset Z-(A \cup B)$ because $Z^{\prime} \cap S=\emptyset$. These results contradict the choice of $Z$.

We conclude that there is no bond $Y$ for which $S \subset Y \subset S \cup \partial u$. Similarly there is no bond $Y$ such that $S \subset Y \subset S \cup \partial w$.

\section{Corollary 21.}

1. $u$ and $w$ are vertices of $G$.

2. $Z-\partial u-\partial w$ is a bond of $H-\{u, w\}$.

Proof. As a consequence of Lemma 20, there is no $S$ such that $G<A \cup B \cup S>$ may be obtained from $G<A \cup B>$ by splitting the vertex $u$ or $w$. Hence, $u$ and $w$ are vertices of G.

Clearly, $Z-\partial u-\partial w$ is a cocycle $[T, V H-\{u, w\}-T]$ of $H-\{u, w\}$. Both $[T,(V H-$ $T) \cup\{u, w\}]$ and $[T \cup\{u, w\}, V H-\{u, w\}-T]$ are among the bonds $Z, Z+\partial u, Z+\partial w$, and $Z+\partial u+\partial w$. Hence, both $H[T]$ and $H[V H-\{u, w\}-T]$ are connected so that $Z-\partial u-\partial w$ is a bond.

We suppose that $v$ is the vertex of $G<A \cup B>$ that is split in the formation of $S$, and let it be split into vertices $v^{\prime}$ and $v^{\prime \prime}$ so that $S$ is the rope $\left[v^{\prime}, v^{\prime \prime}\right]$ in $G<A \cup B \cup S>$ and so that $R$ is contained in $\left[x, v^{\prime \prime}\right]$.

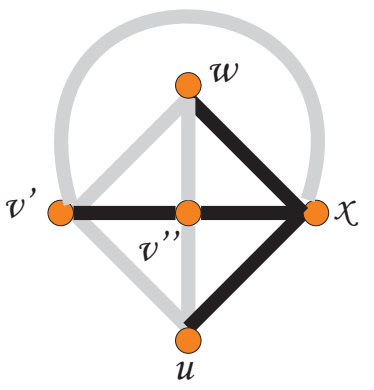

Figure 5: The graph $G<A \cup B \cup S>$. In this and the subsequent figures, ropes which are known to exist are drawn in black. Further ropes which might exist are drawn in grey.

By construction, $G<A \cup B \cup S>$ has ropes $[u, x],[w, x],\left[x, v^{\prime \prime}\right]$ and $\left[v^{\prime}, v^{\prime \prime}\right]$, while $[u, w]$ is empty and thus not a rope. Further, there is at least one rope of $G<A \cup B \cup S>$ in each of the following sets: $\left\{\left[u, v^{\prime}\right],\left[u, v^{\prime \prime}\right]\right\},\left\{\left[w, v^{\prime}\right],\left[w, v^{\prime \prime}\right]\right\}$, and $\left\{\left[u, v^{\prime}\right],\left[w, v^{\prime}\right],\left[x, v^{\prime}\right]\right\}$. By Lemma 20 and on considering the bond $\partial v^{\prime}$ of $G<A \cup B \cup S>$, we further conclude that there is at least is at least one rope of $G<A \cup B \cup S>$ in each of the sets $\left\{\left[u, v^{\prime}\right],\left[x, v^{\prime}\right]\right\}$ and $\left\{\left[w, v^{\prime}\right],\left[x, v^{\prime}\right]\right\}$. 
Lemma 22. The following four statements are equivalent:

1. $G=G<A \cup B \cup S>$.

2. $Z$ is one of $\partial v^{\prime}, \partial\left\{u, v^{\prime}\right\}, \partial\left\{w, v^{\prime}\right\}$ or $\partial\left\{x, v^{\prime \prime}\right\}$.

3. $Z$ includes $\left[x, v^{\prime}\right]$.

4. Both $\left[u, v^{\prime}\right]$ and $\left[w, v^{\prime}\right]$ are ropes of $G<A \cup B \cup S>$.

Proof. Suppose $G=G<A \cup B \cup S>$. $Z$ is a bond of $H$ that contains $S$ and meets $\partial u$ and $\partial w$. The only candidates for $Z$ are the cocycles $\partial v^{\prime}, \partial v^{\prime}+\partial u=\partial\left\{u, v^{\prime}\right\}, \partial v^{\prime}+$ $\partial w=\partial\left\{w, v^{\prime}\right\}$ and $\partial v^{\prime}+\partial u+\partial w=\partial\left\{x, v^{\prime \prime}\right\}$. The converse follows immediately from Corollary 19.

Moreover, if $Z$ is one of $\partial v^{\prime}, \partial\left\{u, v^{\prime}\right\}, \partial\left\{w, v^{\prime}\right\}$ or $\partial\left\{x, v^{\prime \prime}\right\}$ then it includes $\left[x, v^{\prime}\right]$. On the other hand, assume $Z$ includes $\left[x, v^{\prime}\right]$. By Corollary 21, $Z-\partial u-\partial w$ is a bond of $H-\{u, w\}$, and therefore equals $S \cup\left[x, v^{\prime}\right]=\left[v^{\prime},\left\{x, v^{\prime \prime}\right\}\right]$. Hence, $Z$ is one of $\partial v^{\prime}, \partial\left\{u, v^{\prime}\right\}$, $\partial\left\{w, v^{\prime}\right\}$ or $\partial\left\{x, v^{\prime \prime}\right\}$.

Finally, it is easy to check that $\left[u, v^{\prime}\right]$ and $\left[w, v^{\prime}\right]$ are ropes of $G<A \cup B \cup S>$ if $\partial v^{\prime}$ is a bond of $H$ that meets $\partial u$ and $\partial w$. A similar statement holds for $\partial\left\{u, v^{\prime}\right\}, \partial\left\{w, v^{\prime}\right\}$ and $\partial\left\{x, v^{\prime \prime}\right\}$. Conversely, if $\left[u, v^{\prime}\right]$ and $\left[w, v^{\prime}\right]$ are ropes of $G<A \cup B \cup S>$, then $G=$ $G<A \cup B \cup S>$ by the minimality of $Z$ since $\partial v^{\prime}$ is a bond of $H$ that meets $\partial u$, $\partial w$, and $S$.

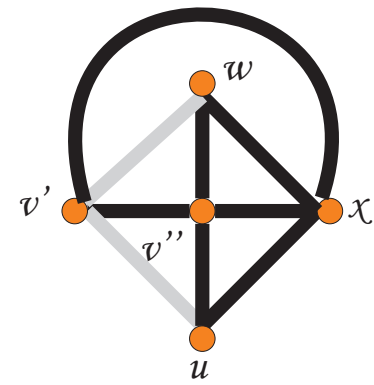

Figure 6: The graph $G<A \cup B \cup S>$ in the case that $\left[x, v^{\prime}\right],\left[u, v^{\prime \prime}\right]$, and $\left[w, v^{\prime \prime}\right]$ are ropes.

Lemma 23. If $\left[x, v^{\prime}\right],\left[u, v^{\prime \prime}\right]$, and $\left[w, v^{\prime \prime}\right]$ are ropes of $G<A \cup B \cup S>$, then $G=$ $G<A \cup B \cup S>$.

Proof. Let $Y_{1}$ and $Y_{2}$ be the two even bonds among $\partial v^{\prime \prime}, \partial v^{\prime \prime}+\partial u=\partial\left\{u, v^{\prime \prime}\right\}, \partial v^{\prime \prime}+\partial w=$ $\partial\left\{w, v^{\prime \prime}\right\}$ and $\partial v^{\prime \prime}+\partial u+\partial w=\partial\left\{x, v^{\prime}\right\}$. It is easy to check that $A+B=\partial u+\partial w=Y_{1}+Y_{2}$ and $A \cup B \cup Y_{1} \cup Y_{2}=A \cup B \cup S$. If $\left\{A, B, Y_{1}, Y_{2}\right\}$ is a $J$-intractable set of even bonds of $G$ then the lemma holds by the minimality of $G$. Suppose therefore that $\left\{A, B, Y_{1}, Y_{2}\right\}$ is not $J$-intractable. Hence, exactly one of $Y_{1}$ and $Y_{2}$ has the directed parity assigned by $J$. Then, however, $\left\{Y_{1}, Y_{2}, Z_{1}, Z_{2}\right\}$ is a $J$-intractable set of even bonds of $G$, and $G=$ 
$G<Y_{1} \cup Y_{2} \cup Z_{1} \cup Z_{2}>$. Neither $Y_{1}$ nor $Y_{2}$ includes the rope $\left[x, v^{\prime}\right]$ so that $Z_{1} \cup Z_{2}$ includes $\left[x, v^{\prime}\right]$. This implies that $Z$ includes $\left[x, v^{\prime}\right]$ and concludes the proof by Lemma 22 .

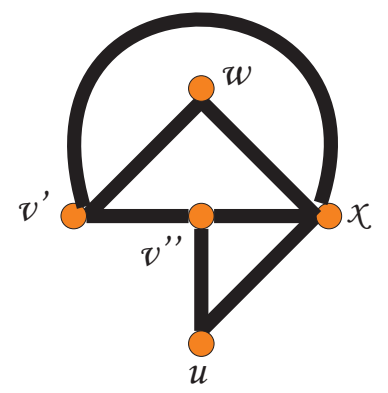

Figure 7: The graph $G<A \cup B \cup S>$ in the case that $\left[u, v^{\prime}\right]$ is not a rope.

Lemma 24. $G=G<A \cup B \cup S>$.

Proof. By Lemma 22 it suffices to show that both $\left[u, v^{\prime}\right]$ and $\left[w, v^{\prime}\right]$ are ropes of $G<A \cup B \cup S>$. Suppose $\left[u, v^{\prime}\right]$ is empty, that is, not a rope of $G<A \cup B \cup S>$. We immediately conclude that $\left[u, v^{\prime \prime}\right]$ and $\left[x, v^{\prime}\right]$ are ropes of $G<A \cup B \cup S>$. If $\left[w, v^{\prime \prime}\right]$ is a rope of $G<A \cup B \cup S>$ then the lemma follows from Lemma 23. Suppose therefore that $\left[w, v^{\prime \prime}\right]$ is not a rope. Hence $\left[w, v^{\prime}\right]$ is a rope. (See Figure 7.) Note that both $\partial v^{\prime \prime}$ and $\partial\left\{u, v^{\prime \prime}\right\}$ are bonds of $G<A \cup B \cup S>$, and both include $\left[x, v^{\prime \prime}\right]$ and thus $R$. One of them, say $C$, is an even bond and thus a possible replacement for $A$ or $B$ in the initial choice of even bonds $A$ and $B$. Recall that $A$ and $B$ are either $\partial x$ and $\partial v=\partial\left\{v^{\prime}, v^{\prime \prime}\right\}$, or $\partial\{w, x\}$ and $\partial\{u, x\}$.

Suppose $A$ and $B$ are $\partial x$ and $\partial v$. Further suppose that among $C$ and $\partial x$ just one has the directed parity prescribed by $J$. Then by assumption and Remark $17, \partial x+C$ is the sum of two disjoint odd bonds. But if $\partial v^{\prime \prime}$ is odd, then $C=\partial\left\{u, v^{\prime \prime}\right\}$, and $\partial x+C=\partial\left\{w, v^{\prime}\right\}$ is a bond itself. Therefore $\partial v^{\prime \prime}$ must be even, so that $C=\partial v^{\prime \prime}$. We observe that $\partial x \cap C$ is a proper subset of $A \cap B=\partial x \cap \partial\left\{v^{\prime}, v^{\prime \prime}\right\}$ as $\left[x, v^{\prime}\right]$ is non-empty. This contradicts the minimality of $A \cap B$.

Otherwise, among $C$ and $\partial v$ just one has the directed parity prescribed by $J$. Then by assumption $C+\partial v$ is the sum of two disjoint odd bonds. But if $\partial v^{\prime \prime}$ is even, then $C=\partial v^{\prime \prime}$, and $C+\partial v=\partial v^{\prime}$ is a bond itself. Therefore $\partial v^{\prime \prime}$ must be odd, so that $C=\partial\left\{u, v^{\prime \prime}\right\}$. We observe that $C \cap \partial v$ is a proper subset of $A \cap B=\partial x \cap \partial\left\{v^{\prime}, v^{\prime \prime}\right\}$ as $\left[x, v^{\prime}\right]$ is non-empty. This contradicts the minimality of $A \cap B$.

Now, suppose $A$ and $B$ are $\partial\{w, x\}$ and $\partial\{u, x\}$. Further suppose that among $C$ and $\partial\{w, x\}$ just one has the directed parity prescribed by $J$. Then by assumption $\partial\{w, x\}+C$ is the sum of two disjoint odd bonds. But if $\partial v^{\prime \prime}$ is odd, then $C=\partial\left\{u, v^{\prime \prime}\right\}$, and $\partial\{w, x\}+C=\partial v^{\prime}$ is a bond itself. Therefore $\partial v^{\prime \prime}$ must be even, so that $C=\partial v^{\prime \prime}$. We observe that $\partial\{w, x\} \cap C$ is a proper subset of $A \cap B=\partial x \cap \partial\left\{v^{\prime}, v^{\prime \prime}\right\}$ as $\left[x, v^{\prime}\right]$ is non-empty. This contradicts the minimality of $A \cap B$. 
Otherwise, among $C$ and $\partial\{u, x\}$ just one has the directed parity prescribed by $J$. Then by assumption $C+\partial\{u, x\}$ is the sum of two disjoint odd bonds. But if $\partial v^{\prime \prime}$ is even, then $C=\partial v^{\prime \prime}$, and $C+\partial\{u, x\}=\partial\left\{w, v^{\prime}\right\}$ is a bond itself. Therefore $\partial v^{\prime \prime}$ must be odd, so that $C=\partial\left\{u, v^{\prime \prime}\right\}$. We observe that $C \cap \partial\{u, x\}$ is a proper subset of $A \cap B=\partial x \cap \partial\left\{v^{\prime}, v^{\prime \prime}\right\}$ as $\left[x, v^{\prime}\right]$ is non-empty. This contradicts the minimality of $A \cap B$.

In all cases we have derived a contradiction. Hence, $\left[u, v^{\prime}\right]$ cannot be empty, but must be a rope of $G<A \cup B \cup S>$. Similarly, we may prove that $\left[w, v^{\prime}\right]$ is a rope of $G<A \cup B \cup S>$. By Lemma 22 this concludes the proof.

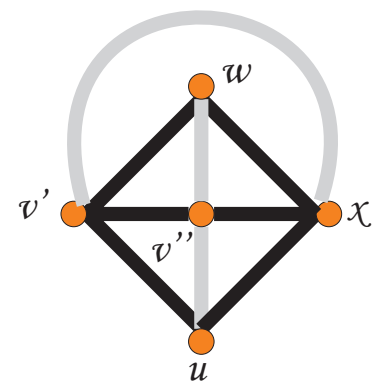

Figure 8: The graph $G<A \cup B \cup S>$ with the ropes $\left[u, v^{\prime}\right]$ and $\left[w, v^{\prime}\right]$ which are now known to exist.

Lemma 25. $\partial v^{\prime \prime}$ is odd.

Proof. Assume that $\partial v^{\prime \prime}$ is even. Suppose $A$ and $B$ are $\partial x$ and $\partial v=\partial\left\{v^{\prime}, v^{\prime \prime}\right\}$. Consider the sets $\left\{A, \partial v^{\prime \prime}, A+\partial v^{\prime \prime}\right\}$ and $\left\{B, \partial v^{\prime \prime}, B+\partial v^{\prime \prime}\right\}$ of even bonds of $G$, that is, the sets $\left\{\partial x, \partial v^{\prime \prime}, \partial\left\{x, v^{\prime \prime}\right\}\right\}$ and $\left\{\partial\left\{v^{\prime}, v^{\prime \prime}\right\}, \partial v^{\prime \prime}, \partial v^{\prime}\right\}$. One of them is a $J$-intractable set in $G$. But $\partial x \cup \partial v^{\prime \prime}$ is a proper subset of $A \cup B \cup S$ as $\left[u, v^{\prime}\right]$ is non-empty. Similarly, $\partial v \cup \partial v^{\prime \prime}$ is a proper subset of $A \cup B \cup S$ as $[u, x]$ is non-empty.

Now, suppose $A$ and $B$ are $\partial\{w, x\}$ and $\partial\{u, x\}$. Consider the sets $\left\{A, \partial v^{\prime \prime}, A+\partial v^{\prime \prime}\right\}$ and $\left\{B, \partial v^{\prime \prime}, B+\partial v^{\prime \prime}\right\}$ of even bonds of $G$, that is, the sets $\left\{\partial\{w, x\}, \partial v^{\prime \prime}, \partial\left\{u, v^{\prime}\right\}\right\}$ and $\left\{\partial\{u, x\}, \partial v^{\prime \prime}, \partial\left\{w, v^{\prime}\right\}\right\}$. One of them is a $J$-intractable set in $G$. But $\partial\{w, x\} \cup \partial v^{\prime \prime}$ is a proper subset of $A \cup B \cup S$ as $\left[u, v^{\prime}\right]$ is non-empty. Similarly, $\partial\{u, x\} \cup \partial v^{\prime \prime}$ is a proper subset of $A \cup B \cup S$ as $[u, x]$ is non-empty.

In both cases we have found a $J$-intractable set of even bonds of $G<A \cup B \cup S>$ such that the union of these bonds is a proper subset of $A \cup B \cup S$. This contradicts the fact that $G$ is minimally $J$-nonorientable. Hence we may conclude that $\partial v^{\prime \prime}$ is odd.

Lemma 26. Neither $\left[u, v^{\prime \prime}\right]$ nor $\left[w, v^{\prime \prime}\right]$ is a rope of $G=G<A \cup B \cup S>$.

Proof. Assume $\left[w, v^{\prime \prime}\right]$ is non-empty. Suppose $A$ and $B$ are $\partial x$ and $\partial v=\partial\left\{v^{\prime}, v^{\prime \prime}\right\}$. Consider the sets $\left\{A, \partial\left\{w, v^{\prime \prime}\right\}, A+\partial\left\{w, v^{\prime \prime}\right\}\right\}$ and $\left\{B, \partial\left\{w, v^{\prime \prime}\right\}, B+\partial\left\{w, v^{\prime \prime}\right\}\right\}$ of even bonds of $G$, that is, the sets $\left\{\partial x, \partial\left\{w, v^{\prime \prime}\right\}, \partial\left\{u, v^{\prime}\right\}\right\}$ and $\left\{\partial\left\{v^{\prime}, v^{\prime \prime}\right\}, \partial\left\{w, v^{\prime \prime}\right\}, \partial\left\{w, v^{\prime}\right\}\right\}$. One of 
them is a $J$-intractable set in $G$. But $\partial x \cup \partial\left\{w, v^{\prime \prime}\right\}$ is a proper subset of $A \cup B \cup S$ as $\left[u, v^{\prime}\right]$ is non-empty. Similarly, $\partial v \cup \partial\left\{w, v^{\prime \prime}\right\}$ is a proper subset of $A \cup B \cup S$ as $[u, x]$ is non-empty.

Now, suppose $A$ and $B$ are $\partial\{w, x\}$ and $\partial\{u, x\}$. Consider the sets $\left\{A, \partial\left\{w, v^{\prime \prime}\right\}, A+\right.$ $\left.\partial\left\{w, v^{\prime \prime}\right\}\right\}$ and $\left\{B, \partial\left\{w, v^{\prime \prime}\right\}, B+\partial\left\{w, v^{\prime \prime}\right\}\right\}$ of even bonds of $G$, that is, the sets $\left\{\partial\{w, x\}, \partial\left\{w, v^{\prime \prime}\right\}, \partial\left\{x, v^{\prime \prime}\right\}\right\}$ and $\left\{\partial\{u, x\}, \partial\left\{w, v^{\prime \prime}\right\}, \partial v^{\prime}\right\}$. One of them is a $J$-intractable set in $G$. But $\partial\{w, x\} \cup \partial\left\{w, v^{\prime \prime}\right\}$ is a proper subset of $A \cup B \cup S$ as $\left[u, v^{\prime}\right]$ is non-empty. Similarly, $\partial\{u, x\} \cup \partial\left\{w, v^{\prime \prime}\right\}$ is a proper subset of $A \cup B \cup S$ as $[u, x]$ is non-empty.

In both cases we have found a $J$-intractable set of even bonds of $G=G<A \cup B \cup S>$ such that the union of these bonds is a proper subset of $A \cup B \cup S$. This contradicts the fact that $G=G<A \cup B \cup S>$ is minimally $J$-nonorientable. Hence we may conclude that $\left[w, v^{\prime \prime}\right]$ is empty, that is, not a rope of $G$. Similarly, we may disprove the existence of a rope $\left[u, v^{\prime \prime}\right]$ of $G$.

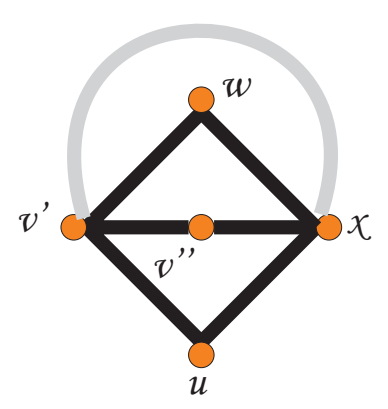

Figure 9: The graph $G=G<A \cup B \cup S>$ without the ropes $\left[u, v^{\prime \prime}\right]$ and $\left[w, v^{\prime \prime}\right]$ which are now known not to exist.

Corollary 27. $G=G<A \cup B \cup S>$ is an even duplication of one of the graphs in Figure 1.

Proof. By Lemma 16 and the proper vertex splitting of $v, G=G<A \cup B \cup S>$ has ropes $[u, x],[w, x],\left[x, v^{\prime \prime}\right]$ and $\left[v^{\prime}, v^{\prime \prime}\right]$, while $[u, w]$ was empty and thus not a rope. By Lemma 24, $\left[u, v^{\prime}\right]$ and $\left[w, v^{\prime}\right]$ are ropes of $G$, while by Lemma 26, $\left[u, v^{\prime \prime}\right]$ and $\left[w, v^{\prime \prime}\right]$ are empty and thus not ropes of $G$. Finally, $\left[x, v^{\prime}\right]$ may or may not be a rope of $G$.

Also, by Lemma 16, $u$ and $w$ have odd degree, and by Lemma 25, $v^{\prime \prime}$ has odd degree. The remaining vertices, $x$ and $v^{\prime}$ both may have even or odd degree. It is an easy exercise to check that, due to these properties, the graph $G$ is an even duplication of one of the graphs in Figure 1.

By the assumptions at the beginning of this section and by Corollary 27, we finally derive Theorem 1, the main result of this paper. 


\section{Proof of Lemma 16}

It remains to prove Lemma 16 stating that the even bonds $A$ and $B$ can be chosen so that $G<A \cup B>$ is an even duplication of a graph in Figure 3.

Proof. We assume that $A$ and $B$ have been chosen with the properties above so that $A \cup B$ is minimal. We may assume without loss of generality that $A$ has the directed parity prescribed by $J$. Clearly, $A$ and $B$ are even bonds of $F=G<A \cup B>$, and thus $A+B$ is a cocycle of $F$. Say $A=\partial S$ and $B=\partial T$ in $F$. Let $R$ connect two vertices $x \in S-T$ and $y \in T-S$ in $F$.

It should be noted that each of $S \cap T, S-T, T-S$ and $V F-S-T$ is an independent set of $F$, while each of $F[S], F[T], F[V F-S]$ and $F[V F-T]$ is connected. Consequently every vertex in $S$ has a neighbour in $V F-S$.

First we find a subset $X$ of $S$ with $x \in X$ and such that $F[X]$ is connected and $\partial X$ has even parity in $F$. If $x$ has even degree in $F$ we choose $X=\{x\}$. Otherwise, $S$ contains vertices with odd degree in $F$ other than $x$ because $\partial S$ is even. We may choose $X$ as the vertex set of a shortest path from $x$ to any of these vertices. Note that $X$ is a proper subset of $S$, unless $X=S=\{x\}$ or $F[S]$ itself is a path from $x$ to some vertex $x^{\prime}$ where $x^{\prime}$ is the only other vertex in $S$ whose degree in $F$ is odd. We are going to construct a new even bond $B^{\prime} \neq A$ in $F$ containing $R$ in each of the cases. But first we show that any such $B^{\prime}$ has the directed parity prescribed by $J$. Suppose the contrary. By $B^{\prime} \cup B \subseteq A \cup B$ and the minimality of $A \cup B$ we obtain $B^{\prime} \cup B=A \cup B$ and therefore $A+B^{\prime} \subseteq B$. Furthermore, since $A \neq B^{\prime}$, it follows that $A+B^{\prime}$ is a non-empty cocycle and thus equals $B$. This contradicts $R \subseteq B$.

Case 1: Firstly suppose we have $X=S=\{x\}$. Then $S-T=\{x\}, S \cap T$ is empty, $T-S=\{y\}$ since $B$ is a bond, and every vertex in $V F-S-T$ is adjacent to $x$ and to $y$. Among the vertices in $V F-S-T$ we find one, say $z$, of even degree, or we find two, say $z_{1}$ and $z_{2}$, of odd degree. This is because $\partial(V F-S-T)=A+B$ has even parity. In the first subcase, put $T^{\prime}=V F-\{x, z\}$, and $B^{\prime}=\partial T^{\prime}$. Clearly, $F\left[T^{\prime}\right]$ is connected, and so is $F\left[V F-T^{\prime}\right]=F[\{x, z\}]$. In the second subcase, put $T^{\prime}=V F-\left\{x, z_{1}, z_{2}\right\}$, and $B^{\prime}=\partial T^{\prime}$. Clearly, $F\left[T^{\prime}\right]$ is connected, and so is $F\left[V F-T^{\prime}\right]=F\left[\left\{x, z_{1}, z_{2}\right\}\right]$. In both cases, $B^{\prime}$ is an even bond of $F$ and, thus, of $G$.

In the first subcase $G<A \cup B^{\prime}>$ is an Eulerian trigon with vertex set $\{\{x\},\{z\}, V F-$ $\{x, z\}\}$. Its ropes are therefore all even or all odd. In the second subcase $G<A \cup B^{\prime}>$ has vertex set $\left\{\{x\},\left\{z_{1}\right\},\left\{z_{2}\right\}, V F-\left\{x, z_{1}, z_{2}\right\}\right\}$, the only vertices of odd degree being $\left\{z_{1}\right\}$ and $\left\{z_{2}\right\}$. Moreover, $\left\{z_{1}\right\}$ and $\left\{z_{2}\right\}$ are the only vertices not joined to each other by a rope. We may assume without loss of generality that the rope $\left[\{x\},\left\{z_{1}\right\}\right]$ is odd. There are then two possible solutions: the set of even ropes is either $\left\{\left[\left\{z_{1}\right\}, V F-\left\{x, z_{1}, z_{2}\right\}\right],\left[\{x\},\left\{z_{2}\right\}\right]\right\}$ or the set of ropes incident with $V F-\left\{x, z_{1}, z_{2}\right\}$. Both solutions are represented in Figure 3.

Case 2: Suppose next that $X$ is a proper subset of $S$. Every vertex in $V F-X$ belongs to $V F-S$ or has a neighbour in $V F-S$. Hence $F-X$ is connected, and $\partial X$ is an even bond of $F$ and, thus, also of $G$. Now we consider the components of $F[S-X]$. Since $F[S]$ is connected, each of them contains a vertex which has a neighbour in $X$. As pointed 
out before, this vertex has a neighbour in $V F-S$, too. Among all these components we find one, say $C$, where $\partial V C$ has even parity, or we find two, say $C_{1}$ and $C_{2}$, where both $\partial V C_{1}$ and $\partial V C_{2}$ have odd parity. This is because $\partial S$ and $\partial X$ have even parity. Put $T^{\prime}=S-V C$ or $T^{\prime}=S-\left(V C_{1} \cup V C_{2}\right)$, respectively, and put $B^{\prime}=\partial T^{\prime}$. Clearly, $F\left[T^{\prime}\right]$ is connected, and so is $F\left[V F-T^{\prime}\right]$ which is $F[(V F-S) \cup V C]$ or $F\left[(V F-S) \cup V C_{1} \cup V C_{2}\right]$, respectively. Further, the parity of $B^{\prime}$ is just the parity of $\partial S$ minus the parity of $\partial V C$ or minus the sum of the parities of $\partial V C_{1}$ and $\partial V C_{2}$, respectively. Hence $B^{\prime}$ is an even bond of $F$ and, thus, of $G$.

If $T^{\prime}=S-V C$ then $G<A \cup B^{\prime}>$ is an Eulerian trigon with vertex set $\left\{V C, T^{\prime}, V F-S\right\}$. In the remaining subcase it has vertex set $\left\{V C_{1}, V C_{2}, T^{\prime}, V F-S\right\}$. Moreover $V C_{1}$ and $V C_{2}$ are the only vertices of odd degree and the only two vertices not joined by a rope. As in case 1 the possibilities are represented in Figure 3.

Case 3: Suppose finally that $F[S]$ is a path from $x$ to $x^{\prime}$ such that $x$ and $x^{\prime}$ have odd degree in $F$ while every other vertex in $S$ has even degree. We may suppose that $F[V F-S]$ is a path from $y$ to some vertex $y^{\prime}$ such that $y$ and $y^{\prime}$ have odd degree in $F$ while every other vertex in $V F-S$ has even degree. Put $T^{\prime}=(S-\{x\}) \cup\{y\}$ and $B^{\prime}=\partial T^{\prime}$. Since $F[V F-T]$ is connected, $x$ has a neighbour in $V F-S-T \subseteq V F-S-\{y\}$. Hence $F\left[V F-T^{\prime}\right]$ is connected. Similarly, we see that $F\left[T^{\prime}\right]$ is connected. Further, the parity of $B^{\prime}$ is the parity of $A$ minus the parity of the degree of $x$ plus the parity of the degree of $y$. Hence $B^{\prime}$ is an even bond of $F$ and, thus, of $G$.

Note that $G<A \cup B^{\prime}>$ is a graph with vertex set $\{\{x\},\{y\}, S-\{x\}, V F-(S \cup\{y\})\}$. The degree of each vertex is odd. Moreover any two vertices are joined by a rope except possibly for $S-\{x\}$ and $V F-(S \cup\{y\})$. If these two vertices are not adjacent, then we may assume without loss of generality that the rope $[S-\{x\},\{y\}]$ is even so that two solutions are possible: the set of odd ropes is either $\{[\{x\}, S-\{x\}],[\{y\}, V F-(S \cup\{y\})]\}$ or the set of ropes incident on $\{x\}$. In the remaining subcase $G<A \cup B^{\prime}>$ is a duplication of $K_{4}$. One possibility is for all the ropes to be odd. Otherwise we may assume without loss of generality that the ropes $[S-\{x\},\{y\}]$ and $[S-\{x\},\{x\}]$ are even. Then the set of odd ropes is either $\{[S-\{x\}, V F-(S \cup\{y\})],[\{x\},\{y\}]\}$ or the set of ropes incident on $V F-(S \cup\{y\})$.

\section{References}

[1] Berge, C. Graphs. North-Holland, 1985.

[2] Fischer, I., And Little, C. H. C. Even circuits of prescribed clockwise parity. Electron. J. Combin. 10 (2003), R45, 20 pages. 